\title{
Design of Traffic Cone Localization and Angle Measurement System Based on Monocular Camera
}

\author{
Chenjian Du ${ }^{1, a}$ and Shuqiang Gao ${ }^{1, b}$ \\ ${ }^{1}$ Zhengzhou University, NO.100 Kexue Road, High-Tech Development Zone of States, \\ Zhengzhou, China \\ adcj.003@163.com, biesqgao@zzu.edu.cn
}

\begin{abstract}
Keywords: Traffic cone; Monocular camera; Image processing; Position determination; Angle
\end{abstract} measurement

\begin{abstract}
In this paper, a traffic cone localization and angle measurement system is designed from the perspective of digital image processing based on monocular camera. The system can assist the traffic cone cart to automatically identify and locate the traffic cones during the process of collecting traffic cones. The angle of the traffic cone is measured when it is laying down based on the positional relationship of the feature points. In order to verify the reliability of the system, we adjust the images collected by the camera, then we recognize the colors and process the images morphology to determine whether the traffic cones exist in the image, finally we get the real positions and the angles in physical space by geometric relation deductive method on the simulation platform VS2010+OpenCV. The measurement error is within an accept range since its mistakes are all less than $2 \mathrm{~cm}$ during the tests. The experimental results show that the system can identify the traffic cones on the road surface effectively and calculate its position and angle information, which can satisfy the needs of the traffic cone cart.
\end{abstract}

\section{Introduction}

With the accelerated pace of traffic, road maintenance construction requirements as far as possible without affecting the traffic order under the promise. The placement and collection of traffic cones is the necessary work in the process of road construction, artificial placement and collection of traffic cones have a low efficiency, and the surrounding vehicles will also cause security risks to persons, the solution is the implementation of automatic placement and collection ${ }^{[1]}$. The automatic placement process of traffic cones is simple, it only need a mechanical arm to carry traffic cones to the appropriate location from its own storage box. The spacing and direction of traffic cones placed mainly rely on the speed and direction of traffic cone cart. But the automatic collection process of traffic cones is quite complex, because the locations of traffic cones on the road are not fixed relative to the path of traffic cone cart.

At present, the researches on traffic cone identification and localization were rare. The effect of the existing research results for traffic cone cart is not very satisfactory. Such as traffic cone recovery system of the ADDCO company in United States, California AHMCT corporation's traffic cone recycling car in pickup truck platform, traffic cone machine invented by Beijing LIANJIEDA highway maintenance engineering company ${ }^{[2]}$, and automatic traffic cone cart of Henan lofty highway maintenance equipment company. In the process of traffic cone collection, traffic Cone identification and localization of the above productions entirely determined by the route of the driver controlling, drivers' driving attention was greatly dispersed in the process of looking for traffic cones. 
Differ from the research results for the mechanical traffic cone collecting which are accounted by the above, we design a traffic cone image processing and identification system in this paper from the point of digital image-processing. This system can get the position information of the traffic cone by using OpenCV visual library as instrument. This system can assist the traffic cone cart identify and locate the traffic cones automatically in the process of collecting traffic cones, it will enhance the automation performance of traffic cone cart and improve the efficiency of collecting traffic cones.

\section{Traffic Cone Characteristic Analysis and System Project Design}

Traffic Cone Characteristic Analysis. Traffic cone is composed of the base and the cone body. As shown in Fig. 1: the base of traffic cone is a square, circumscribed circle diameter of the square is 0.75 times of the traffic cone height. The traffic cone's body height is $65 \mathrm{~cm}$ with red and white reflective surface, the surface can't peel off from the traffic cone body in normal using and should keep back reflection properties in wet state. The traffic cone's body is a conical shape, the circumscribed circle diameter of the traffic cone's upper surface is $60 \mathrm{~mm}$, and there is an edge smooth round hole with $48 \mathrm{~mm}$ on the traffic cone's upper surface.

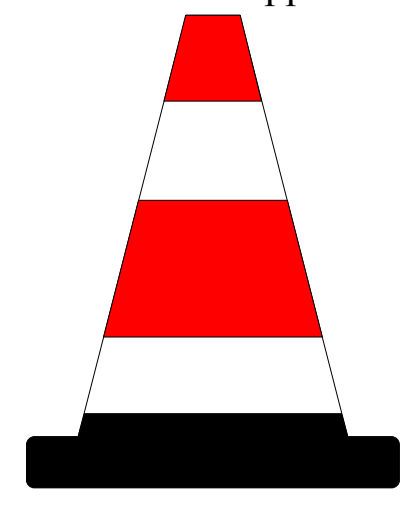

Figure 1. Traffic cone positive vector diagram

We need to analyze the characteristics of the traffic cone: what special features can be extracted from the traffic cone and how are the differences between these features under the condition of different illuminations, then we should manage the features extracted. The traffic cone is a conical shape in three colors: red, white and black, the most significant feature is that it has two red zones whose relative locations are fixed, the feature of the two red zones is easy to distinguish, and the straight view of the feature has two trapezoid up and down. And there are two white trapezoidal zones in close next to the red zones. After analysis we decide to extract the two red zones and the two white zones which are the most obvious of the traffic cone in order to judge whether traffic cones exist in an image or not ${ }^{[3]}$. After extraction we judge the state and the real location of the traffic cone according to the size and the relative location of the two red zones.

Assuming that the first red area is A, the second red area is $\mathrm{B}$, the first white area is $\mathrm{C}$, the second white area is $\mathrm{D}$, the schematic diagram of the traffic cone can be expressed as the following Fig. 2: 


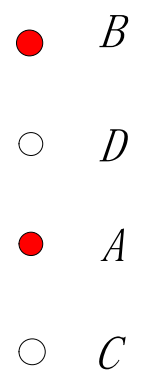

Figure 2. Traffic cone positive simplified diagram

If there is a clear $\mathrm{C}, \mathrm{A}, \mathrm{D}, \mathrm{B}$ arrangement of the position relationship, we can determine the existence of traffic cone. In practical applications, we can find the relative position of traffic cone has a clear relationship in a variety of state.

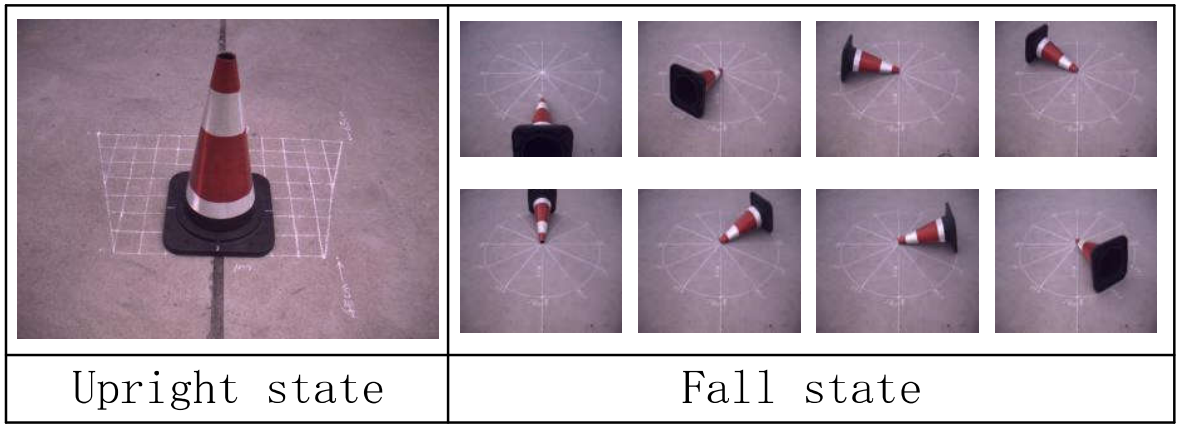

Figure 3. Various states of the traffic cone

The schematic diagram of Fig. 3 above can be shown in Fig. 4.

\begin{tabular}{|c|c|c|c|c|}
\hline - $B$ & $\begin{array}{l}B \\
\bullet \\
\bullet\end{array}$ & $\bullet_{A}^{\bullet B}$ & $\bullet \bullet B$ & $\stackrel{\bullet}{A} B$ \\
\hline - $A$ & $\begin{array}{ll}\bullet & A \\
\bullet & \\
B & \end{array}$ & $\stackrel{\bullet}{\bullet}$ & $B \bullet \bullet$ & $\stackrel{B}{\bullet} \cdot$ \\
\hline Upright state & \multicolumn{4}{|c|}{ Fall state } \\
\hline
\end{tabular}

Figure 4. Simplified states of the traffic cone

Let the coordinates of point $\mathrm{A}$ be $A\left(x_{A}, y_{A}\right)$, and the coordinates of point $\mathrm{B}$ be $B\left(x_{B}, y_{B}\right)$, then the distance between $\mathrm{AB}$ is:

$$
l=\sqrt{\left(y_{B}-y_{A}\right)^{2}+\left(x_{B}-x_{A}\right)^{2}}
$$

The slope between point $\mathrm{B}$ and point $\mathrm{A}$ is

$$
k_{B A}=\frac{y_{B}-y_{A}}{x_{B}-x_{A}}
$$

According to the coordinates of point A, we can calculate the location of the traffic cone. According to the distance between A and B and the slope of geometric relationship, we can judge 
whether the traffic cone is upright or down, and calculate the general direction of the traffic cone when it is in the fall state.

We should take a full consideration of the real conditions such as the illumination and others to make the result having a certain degree of robustness.

System Project Design. The solution architecture design of the traffic cone localization and angle measurement system is shown in Fig. 5. We catch photos of the traffic cones using monocular camera as tool in vision measurement system, then analyze the camera model to get the lens distortion and adjust the mistake. Based on the obvious features in which there are red zones and white zones exist relatively on the traffic cone body, we identify the color of the photo in HSV space and make out the red zones and white zones in it. We take a series of morphological processing with the binary image after color recognition including the object recognition based on connected domain size, contour extraction and confirming the center-of-gravity position of the traffic cone. Under the circumstance that the height and the pitch angle of the camera are fixed, according to the camera and the traffic cone's projection model, adopting the geometric relation deductive method to measure the distance of the traffic cone. Finally the real position of the traffic cone in physical space is obtained.

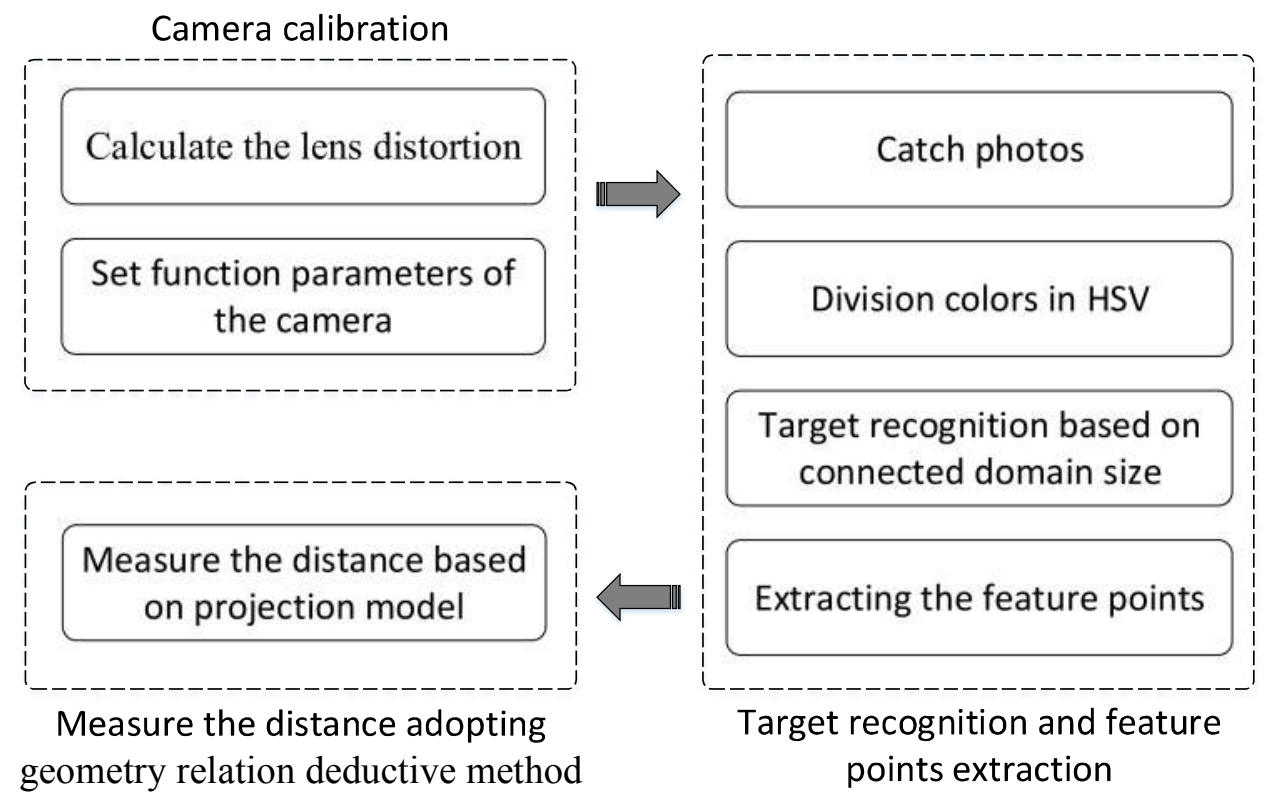

Figure 5. System solution

\section{The Camera Calibration and Distortion Correction}

Always we use pinhole model to do works from the perspective of image processing, but all the type of real cameras will not match the projection transformation of the pinhole model. The father away from the center of the image, the more obvious that image distortion will be. We need correcting the images collected by the camera in order to restore the images to be more authentic and improve the accuracy of the date which is caught after image processing ${ }^{[4]}$.

The Camera Calibration. We use 10 chessboard photos as images for calibration, as shows in Fig. 6. The Fig. 6 on the left shows the locations of the 10 chessboard photos reference for the camera while the Fig. 6 on the right shows the locations of the 10 cameras reference for the chessboard photo. As shown in Fig. 7, it is the No.5 chessboard photo before correcting and after correcting. According to the original images and the corrected image after calibration we can get the distortion coefficients of the camera. 


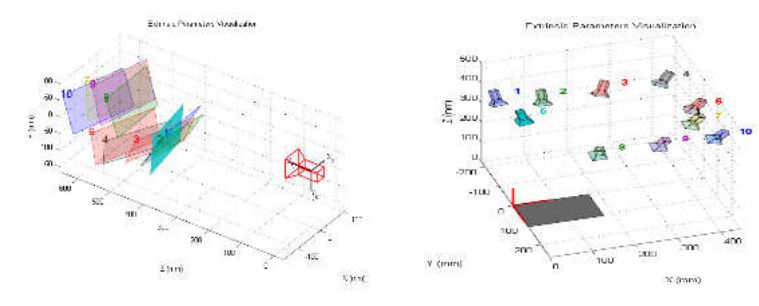

Figure 6. The schematic locations of the calibration images
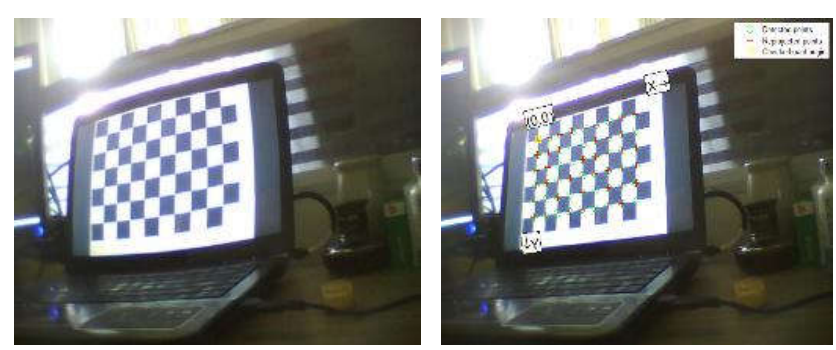

Figure 7. The comparison between the original image and the corrected image after calibration

Distortion Correction. Camera calibration consists of the following steps:

(1) We assume that lens distortion is zero, then we work out the internal parameters and external parameters in a closed form.

(2) Estimate all the parameters including the deformation coefficient at the same time using the nonlinear least squares algorithm (Levenberg-Marquardt). Set the initial deformation coefficient estimating to be zero and use the above steps to get the closed solution as the internal parameters and external parameters of initial estimates ${ }^{[5]}$.
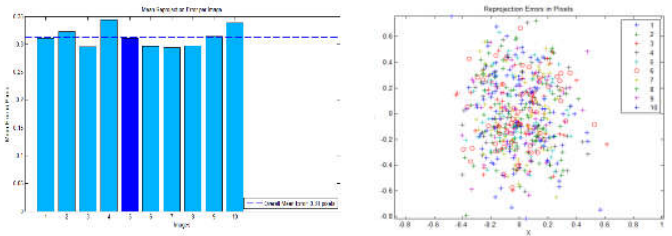

Figure 8 Distortion of the calibration images

The result of the correction for the camera is shown in Fig. 8: finally the lens distortion of the camera is obtained:

Radial Distortion: [-0.1992 0.8068 -2.5421]

Tangential Distortion: [0.0028 3.4989e-04]

\section{The traffic Cone Identification and Feature Point Extraction}

Color recognition. RGB is defined by the color of the human eye to identify the color space, it describes the three primary colors through red, green, and blue. This RGB model is located in the Cartesian coordinate system, as shown in Fig. 9. Red, green and blue of the three-primary colors are located at three corners, secondary colors including cyan, magenta and yellow are located at the other three corners. Any kind of colored light named H can be represented by the Eq. 3

$$
H=r[R]+g[G]+b[B]
$$




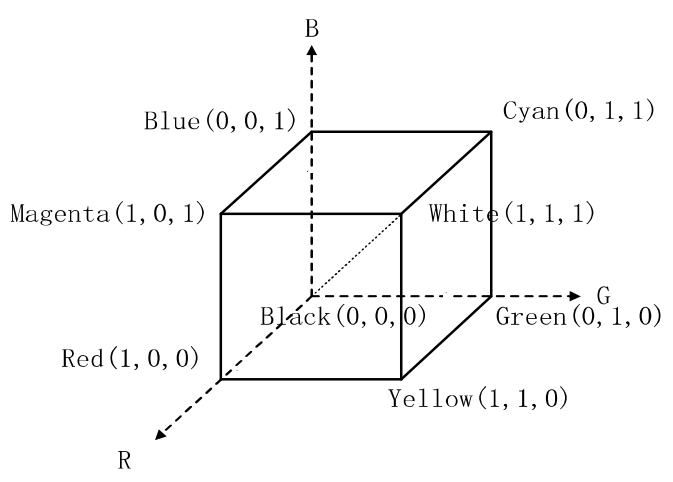

Figure 9. RGB space model

RGB model put the hue, luminance and saturation three components together to show colors, colors are deeply influenced by the luminance. RGB is the most widely used color model face to hardware and it is mainly used in color monitors. In RGB color space, colors are described by all the three components while the three components have a tight relationship. We can't divide any color section only using a single component in RGB color model.

The experimental environment has includes sunny day, cloudy day, the shade, morning, noon, evening and other conditions. The three components will change at the same time as long as the luminance has changed, so the RGB model is not suiChart for image recognition.

The color model of HSV faces to the users, which separates the luminance information and the color information from the images so this method is more consistent with the human eye to describe and explain the colors.

HSV model uses H (Hue), S (Saturation) and V (Value) to show the colors objects. HSV color model is the ideal tool for dealing with color images which can be used to determine the hue of color using only $\mathrm{H}$ component.

HSV color space model is shown in Fig. 10, it is also known as hexagon pyramid model.

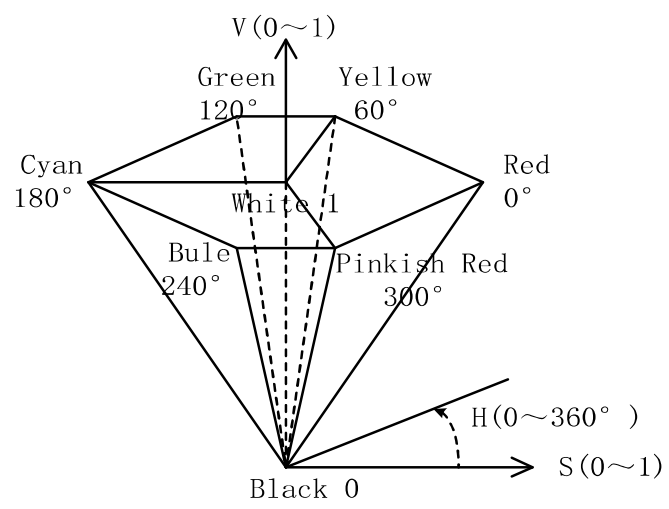

Figure 10. HSV space model

The $\mathrm{H}$ parametric indicates hue and its range is $0^{\circ} \sim 360^{\circ}$, red is $0^{\circ}$, green is $120^{\circ}$ and blue is $240^{\circ}$.

The S parametric indicates saturation indicating the color's degree closed to the spectral color and its range is $0 \sim 1$. The more white-element in a color, the lower the degree of the saturation is.

The V parametric indicates value shown how bright the color is and its range is $0 \sim 1$, but it has no direct relationship with light intensity.

In image processing, we need transferring the colors from RGB space to HSV space in order to process the color recognition: 


$$
\left\{\begin{array}{l}
H_{0}=\operatorname{arcos} \frac{0.5 \times[(R-G)+(R+G)]}{\sqrt{(R-G)^{2}+(R-G)(G-B)}} \\
H= \begin{cases}H_{0} & B \leq G \\
360^{\circ}-H_{0} & B>G\end{cases} \\
S=\frac{\max (R, G, B)-\min (R, G, B)}{\max (R, G, B)} \\
V=\frac{\max (R, G, B)}{255}
\end{array}\right.
$$

In the HSV space, in order to recognize the red zones of the traffic cone we can extract the red pixels from the image through setting the threshold value of $\mathrm{H}$ component as shown in Fig. 11. For the sake of more accurately recognizing the red zones of the traffic cone, we also need setting the threshold values of $\mathrm{S}$ component and $\mathrm{V}$ component. Then we extract each pixel of the image according to the threshold value, and get a binary image after division. The method of recognizing the white zones of the traffic cone is the same as above ${ }^{[6]}$. In the binary image, the region of interest is 1 while the other regions are 0. The binary images we got are shown in Fig. 13 and 14, we judge if there are traffic cones existing in the image as shown in Fig. 12 according to the relationship between the red zones and the white zones.

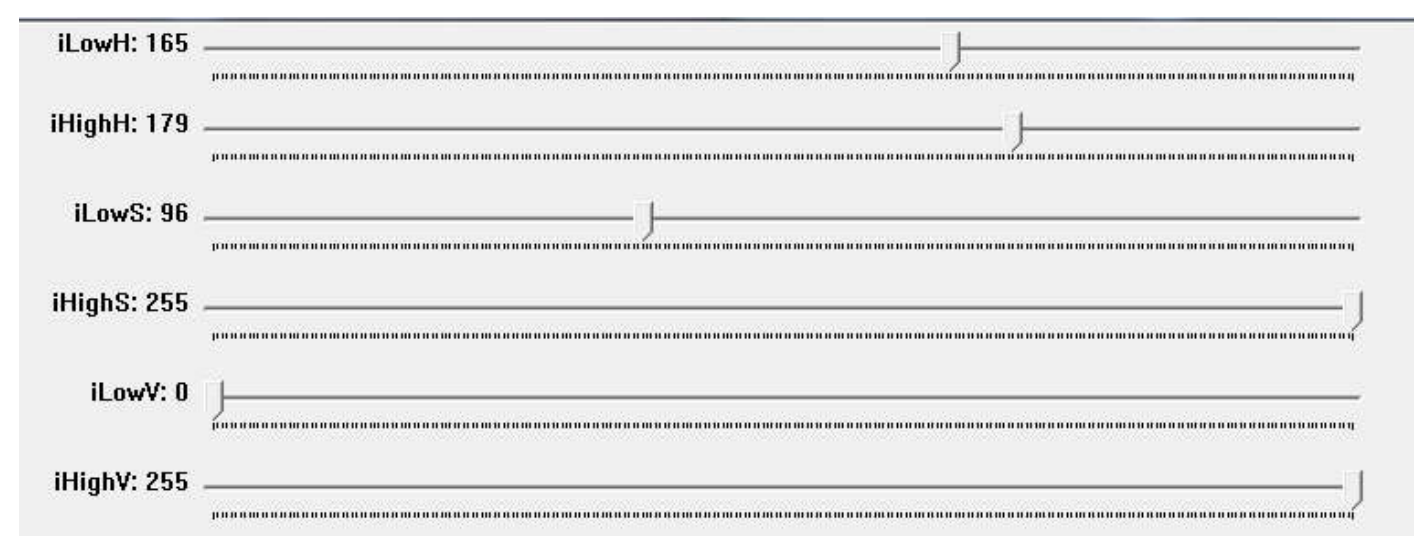

Figure 11. Setting the division threshold value of $\mathrm{H}, \mathrm{S}$ and $\mathrm{V}$ components through the sliders

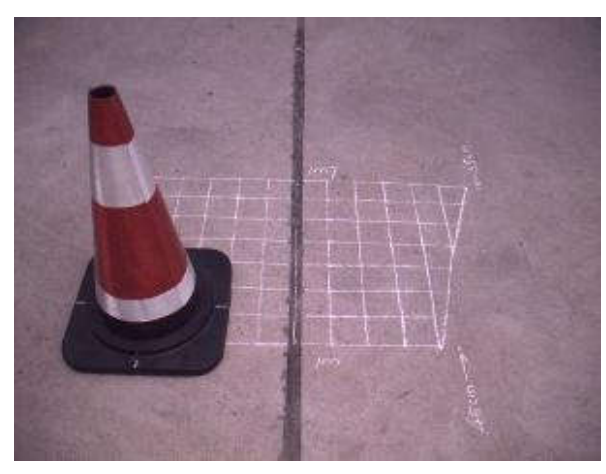

Figure 12. The original image

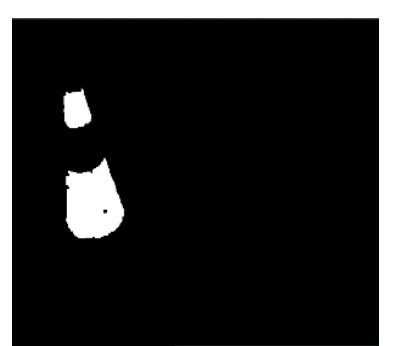

Figure 13. Red zones

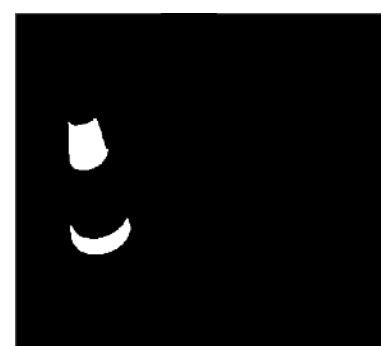

Figure 14. White zones 
Feature Points Extraction. For the image of the traffic cone after extracting the red zones, we can set two threshold values according to the size of the connected domain, and delete any red area which is too large or too small. We want that processed image only having two red zones of the traffic cone.

We let $\mathrm{X}$ be a region and $\mathrm{Z}$ be the boundary of $\mathrm{X}$. Suppose there is an appropriate structural element $\mathrm{Y}$, we use $\mathrm{X}$ to do erosion operation by $\mathrm{Y}$ at first and use $\mathrm{X}$ to do minus operation with the result above at second ${ }^{[7]}$, and $\mathrm{Z}$ can be obtained as shown in Fig. 15-18:

$$
Z=X-(X \ominus Y)
$$

Fig. 15 illustrates the process of boundary extraction. In that process, the structuring element $\mathrm{Y}$ is not the only one to be chose. For example, the size of $Y$ is $5 \times 5$, we will get a boundary with the width of 2 3 pixels. The extracted boundary is the contour of $X$.

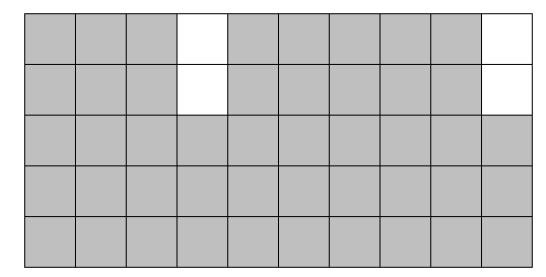

Figure 15. Region X

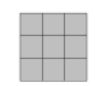

Figure 16. Structuring element $\mathrm{Y}$

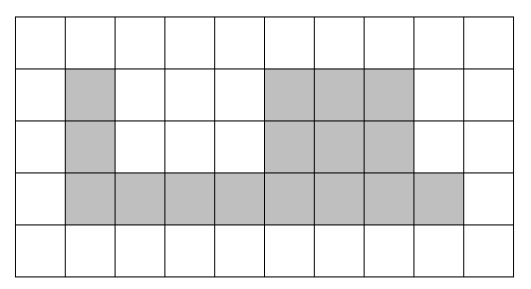

Figure 17. Eroding $\mathrm{X}$ by $\mathrm{Y}$

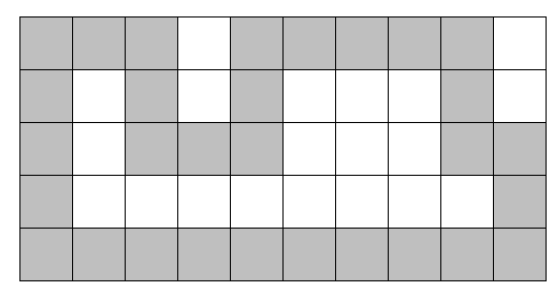

Figure 18. Z: the extracted boundary

After a series of steps such as color recognition and morphological processing, we get the ideal binary image containing two zones (A and $\mathrm{B}$ ) corresponding to the two red zones of the traffic cone as shown in Fig. 17. Next, we extract the boundary of A and B and calculate their bounding rectangles, the two boundary rectangles named $\mathrm{C}$ and $\mathrm{D}$ are obtained ${ }^{[8]}$. $\mathrm{C}$ is the bounding rectangle of the larger red zone and $\mathrm{D}$ is the bounding rectangle of the smaller red zone of the traffic cone. What we are interested in are the four vertices of the rectangle and the barycenter calculating from the four points ${ }^{[9]}$. 
We let $A_{1}$ be the barycenter of $C$ and $A_{2}$ be the barycenter of $D$. The barycenter coordinate are the weighted average of the four points, and the two centers are the feature points of interest we extracted. Among them, $A_{1}$ is used to measure the distance while $A_{1}$ and $A_{2}$ are used to determine the condition of the traffic cone.

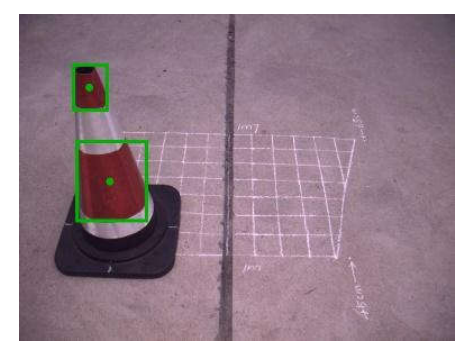

Figure 19. The result of feature points extraction

As shown in Fig. 19, we get the pixel coordinates for the red zones of the traffic cone in the image. The center coordinate of the first red zone: $A_{1}(231,422)$, the center coordinate of the second red zone: $A_{2}(183,209)$.

\section{Traffic cone Location Measurement Model Based on Geometric Relation Deductive Method}

Monocular Vision Ranging Method. The image taken by the camera is a projection from 3D space to $2 \mathrm{D}$ space. The process transforming the $2 \mathrm{D}$ coordinate information in image plane to real location of the object in physical space using geometric relation deductive method called depth information obtaining ${ }^{[10]}$.

This system bases on the principle of pinhole imaging and adopts geometric relation deductive method according to the camera projection model, so this system improves the defects of the corresponding point calibration method to be better and gets the relationship between the world coordinate system and the pixel plane coordinate system, then it realizes the real-time distance measurement.

Actually in OpenCV, the original point of the pixel plane coordinate system is on the top left corner of the image, the positive direction of $\mathrm{X}$ axis is horizontal to the right and the positive direction of $\mathrm{Y}$ axis is vertical downward. But in the image plane coordinate system in this section, the original point is at the center of the image, the positive direction of $\mathrm{X}$ axis is horizontal to the right and the positive direction of $\mathrm{Y}$ axis is vertical upward. The pixel plane coordinate system and the image plane coordinate system need to be converted.

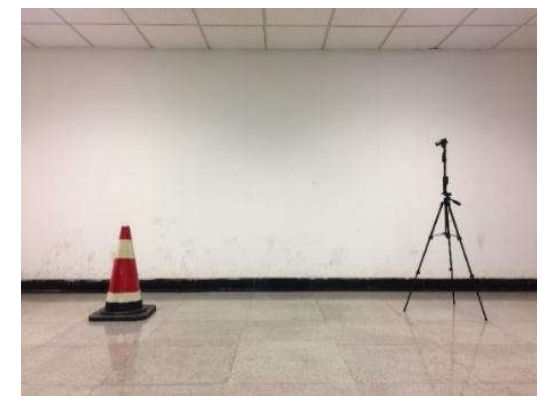

Figure 20. The location of the camera and traffic cone in experiment 
Based on the principle pinhole imaging, the camera projection model in Fig. 20 is shown in Fig. 21-22.

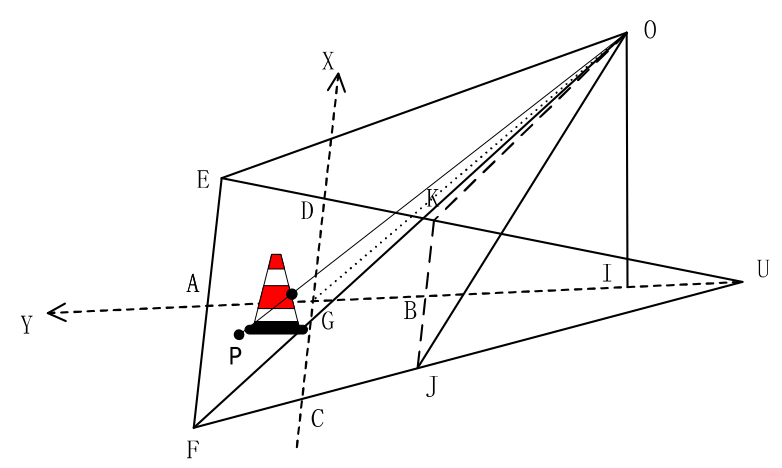

Figure 21. Projection relationship in 3D

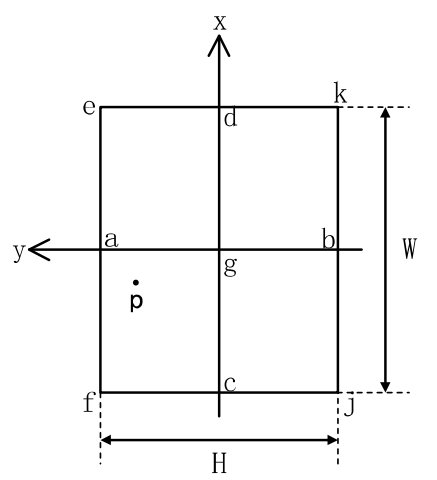

Figure 22. Projection plane in 2D

In Fig. 21, $\mathrm{O}$ is the center point for the lens of the camera and $\mathrm{OG}$ is the optical axis. $\mathrm{G}$ is the intersection of the optical axis and the road surface, and $\mathrm{G}$ is also the intersection of the diagonals for the trapezoid EFJK. EFU is the road surface, and trapezoid EFJK is the range of the road surface where can be shot by the camera. Y axis is the direction where the camera moving toward to and $\mathrm{X}$ axis verticals that forward direction. $G$ is the original point of the road surface coordinate. $I$ is the projection point of lens center $\mathrm{O}$ on the road surface.

Fig. 22 is the image plane corresponding to the road surface shot by the camera. In Fig. 22, e, f, j and $\mathrm{k}$ are the four corners of image plane rectangle corresponding to the $\mathrm{E}, \mathrm{F}, \mathrm{J}$ and $\mathrm{K}$ in Fig. 21, $\mathrm{g}$ is the original point of the coordinate corresponding to $\mathrm{G}$ in Fig. 21. W and $\mathrm{H}$ represent the width and the height of the image plane.

$\mathrm{P}$ on the road surface corresponds to point $\mathrm{p}$ on the image plane. The coordinate of $\mathrm{P}$ in the road surface coordinate system is $\left(X_{P}, Y_{P}\right)$ while the coordinate of point $\mathrm{p}$ in image plane coordinate system is $\left(x_{p}, y_{p}\right)$. We must pay attention to that the coordinate of $\mathrm{P}$ on the road surface coordinate system is not the coordinate of the traffic cone, $\mathrm{P}$ is the intersection of the road surface and the straight line where the center point of the first red zone and the center point of the camera lens in. 


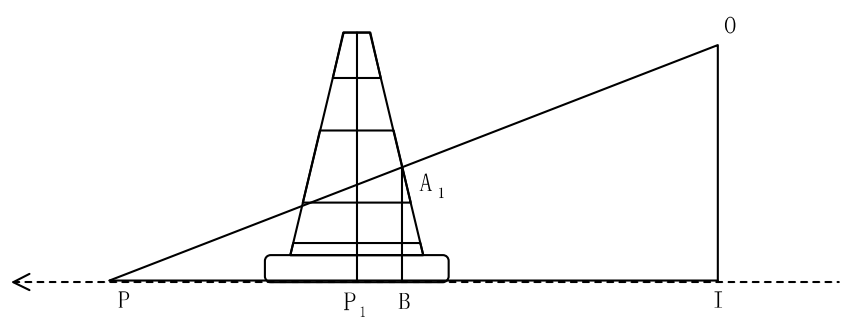

Figure 23. Projection model of the traffic cone

In Fig. 23, we let $A_{1}$ be the center point of the first red zone abstractly, the length of $A_{1} B$ is also the height of point $A_{1}$. $O$ is the center point for the lens of the camera and $I$ is the projection point on the road surface of lens center $\mathrm{O}$. P is the intersection of the road surface and the straight line $\mathrm{OA}_{1}$, actually the coordinate of the traffic cone is point $\mathrm{P}_{1}$. Therefore we need transforming the coordinate of $\mathrm{P}$ to the coordinate of $\mathrm{P}_{1}$ in order to measure the location of a cube with height. According to the similar triangle we can available the Eq. 5:

$$
\frac{A_{1} B}{I O}=\frac{B P}{I P}
$$

Based on Eq. 5 we can get:

$$
I P_{1}=I P \cdot\left(1-\frac{A B}{I O}\right)+B P_{1}
$$

Taking advantage of Fig. 21-23 we can catch the relationship between the image plane coordinate and the road surface coordinate according to the geometric relation deductive method:

$$
\left\{\begin{array}{c}
Y_{P_{1}}=h \cdot \mu_{1} \cdot y_{p} \cdot \frac{1+\mu_{2}^{2}}{1-\mu_{2} \cdot \mu_{1} \cdot y_{p}} \cdot\left(1-\frac{A B}{I O}\right)+B P_{1} \\
X_{P_{1}}=\frac{U G+Y_{P}}{U G} \cdot \mu_{3} \cdot \mu_{4} \cdot x_{p} \cdot\left(1-\frac{A B}{I O}\right)+B P_{1}
\end{array}\right.
$$

The elements in Eq. 7 are shown in Eq. 8:

$$
\left\{\begin{array}{l}
\mu_{1}=\frac{2 \cdot \tan \omega_{0}}{H} \\
\mu_{2}=\tan \theta_{0} \\
\mu_{3}=\frac{h}{\cos \theta_{0}} \\
\mu_{4}=\frac{2 \cdot \tan \lambda_{0}}{W} \\
U G=\frac{h \cdot\left(\tan \theta_{0}-\operatorname{tg}\left(\theta_{0}-\omega_{0}\right)\right) \cdot \cos \left(\theta_{0}-\omega_{0}\right)}{\cos \left(\theta_{0}-\omega_{0}\right)-\cos \theta_{0}}
\end{array}\right.
$$


In Eq. 7 and 8, $\mathrm{h}$ is the height of the camera, $\mathrm{W}$ and $\mathrm{H}$ are the width and the height of the image, $2 \omega_{0}$ and $2 \lambda_{0}$ are the vertical angle and horizontal angle of the view for the camera lens, $\theta_{0}$ is the pitch angle of the camera.

According to the pixel coordinate of the traffic cone in the image, we calculate the coordinate to be $(-40,109)$ since the real coordinate of the traffic cone is $(-40,110)$ in the world coordinate system with the camera as the center point, the result is slightly different.

Angle Measurement. Two feature points $A_{1}$ and $A_{2}$ have been extracted by the extraction algorithm of feature points based on contours. According to the positional relationship between $A_{1}$ and $A_{2}$, the slope of the straight line $A_{1} A_{2}$ is calculated, and the direction and angle of the traffic cone are calculated.

In OpenCV, the coordinates of $A_{1}$ and $A_{2}$ in the pixel coordinate system are $A_{1}\left(x_{1}, y_{1}\right)$ and $A_{2}\left(x_{2}, y_{2}\right)$ respectively. The measurement for the direction of the traffic cone when it is down can be calculated directly in the pixel coordinate system, no need to be switched to the three-dimensional coordinate system.

The measurement steps for the direction of the traffic cone are as follows:

(1)When $x_{2}=x_{1}$, if $y_{2}<y_{1}$, then the traffic cone is in the forward direction; if $y_{2}>y_{1}$, the traffic cone is in the backward direction;

(2) When $x_{2} \neq x_{1}$, calculate the slope of the line $A_{1} A_{2}$ :

$k=-\frac{y_{2}-y_{1}}{x_{2}-x_{1}}$

(3) Calculate the angle: angle' $=\arctan \mathrm{k}$, the range of angle' is $-90 \sim 90^{\circ}$;

(4) When $x_{1}<x_{2}$, angle $=90$-angle', traffic cone falls to the right direction;

(5)When $x_{1}>x_{2}$, angle $=-90$-angle', traffic cone falls to the left direction.

In the actual work, the traffic cone image shot by the camera is not a standard trapezoid because the traffic cone is a cube. The two-dimensional image also lacks the depth information of the traffic cone even in the case of perfect ideals, The vertical coordinates of the two feature points are not necessarily equal to each other when the traffic cone falls in the forward direction perpendicularly to the camera; the situation is similar to that of the other angles.

In the binarized image, the shape of the traffic cone's area is uncontrollable, it is just a trapezoidal-like region. The two feature points extracted from the binarized image are not the exact center of gravity coordinates of the two red regions of the traffic cone.

As the three-dimensional road surface will be missing information in the two-dimensional image, as shown in Fig. 24. In the three-dimensional road surface, the traffic cone is falling to the right with $90^{\circ}$ to the camera's forward direction. But in the two-dimensional image, the straight line $A_{1} A_{2}$ of the feature points is with $103^{\circ}$ to the camera's forward direction. 


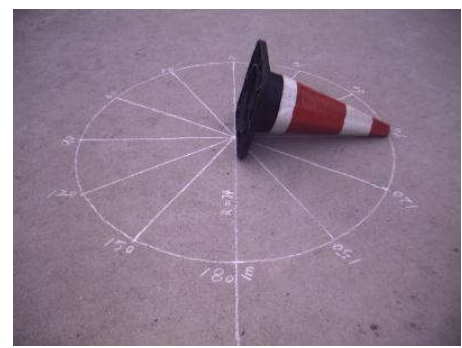

Figure 24. Image of the traffic cone falling down

Therefore, we need to establish a control template library in order to ensure that the measured direction is more accurate. After calculating the direction from the feature points extracted from the two-dimensional image, the mapping of the direction in the actual three-dimensional pavement should be found from the template library.

\section{Experimental Verification and the Results Analysis}

Based on the algorithm for the traffic cone identification and localization including camera calibration and correction, image inputting and transformation, feature points extraction and geometric relation deductive method, we do a large amount of experiments under different light conditions, and the experiments are tried in HASEE Computer(Intel Core i7-2670QM, 4-core CPU, 8GB RAM, 64-bit operating system). We extracted 320 frames containing traffic cones from the videos caught by the camera to recognize and locate, finally 313 images were identified there are traffic cones in them, the identification rate was $97.8 \%$. As shown in Table 1, we get some statistics of the contrasts between the coordinates after calculation and the real coordinates of the traffic cones.

Table 1 The contrasts between the coordinates after calculation and the real coordinates of the traffic cones

\begin{tabular}{lcccccc}
\hline & \multicolumn{2}{c}{ Actual coordinates } & \multicolumn{2}{c}{ Measured coordinates } & \multicolumn{2}{c}{ Deviation } \\
& Number & $\mathrm{X}[\mathrm{cm}]$ & $\begin{array}{c}\mathrm{Y}[\mathrm{c} \\
\mathrm{m}]\end{array}$ & $\begin{array}{c}\mathrm{X}[ \\
\mathrm{cm}]\end{array}$ & $\mathrm{Y}[\mathrm{cm}]$ & $\begin{array}{c}\mathrm{X}[ \\
\mathrm{cm}]\end{array}$ \\
\hline 1 & -40 & 100 & -42 & 100 & -2 & 0 \\
2 & -30 & 100 & -31 & 100 & -1 & 0 \\
3 & -30 & 110 & -31 & 100.5 & -1 & 0.5 \\
4 & -20 & 110 & -20 & 109 & 0 & -1 \\
5 & -20 & 120 & -21 & 119.5 & -1 & -0.5 \\
6 & -10 & 120 & -11 & 121 & -1 & 1 \\
7 & -10 & 130 & -10 & 128.5 & 0 & -1.5 \\
8 & 0 & 130 & -1 & 128.5 & -1 & -1.5 \\
9 & 0 & 140 & -1 & 139 & -1 & -1 \\
10 & 10 & 140 & 9 & 140.5 & -1 & 0.5 \\
11 & 10 & 150 & 8 & 149.5 & -2 & -0.5 \\
12 & 20 & 150 & 18 & 150 & -2 & 0 \\
13 & 20 & 160 & 18 & 161 & -2 & 1 \\
14 & 30 & 160 & 29 & 159 & -1 & -1 \\
15 & 30 & 170 & 30 & 169.5 & 0 & -0.5 \\
\hline
\end{tabular}

Aiming at different light conditions, we adjust the division threshold value for the three components $\mathrm{H}, \mathrm{S}$ and V suitably on the interface, and identify the traffic cones out efficiently. The 
reason why any images can't be recognized is that the traffic cones falls down to the other side contrasting to the camera so that there is no red zone in that images. In consideration of that the center of gravity for the traffic cone is enough low, the traffic cone will not fall down easily. If we exclude this falling factors, the identification rate will be much higher. But we also need exploiting new methods to identify the traffic cones aiming at the extreme falling conditions. The analysis of the experimental result: there is a bit of errors between coordinates after calculation and real coordinates of the traffic cones while the error range is less than $2 \mathrm{~cm}$, and these errors can be accepted if they are in comparison to the size of the traffic cone.

In one test, the angle of the two feature points extracted in the two-dimensional image and the angle in the corresponding three-dimensional pavement are shown in Table 2.

Table 2 Comparison of the same traffic cone in the two-dimensional image and the three-dimensional road surface

\begin{tabular}{|c|c|c|c|}
\hline $\begin{array}{c}\text { The angle in } \\
\text { two - dimensional }\end{array}$ & $\begin{array}{c}\text { The angle in } \\
\text { three-dimensional }\end{array}$ & $\begin{array}{c}\text { The angle in } \\
\text { two - dimensional }\end{array}$ & $\begin{array}{c}\text { The angle in } \\
\text { three-dimensional }\end{array}$ \\
\hline 25 & 20 & -24 & -20 \\
\hline 47 & 40 & -45 & -40 \\
\hline 68 & 60 & -70 & -60 \\
\hline 91 & 80 & -95 & -80 \\
\hline 115 & 100 & -115 & -100 \\
\hline 121 & 120 & -123 & -120 \\
\hline 143 & 140 & -145 & -140 \\
\hline 165 & 160 & -164 & -160 \\
\hline
\end{tabular}

In the template library, we divide the angle directly calculated from the two-dimensional image into several regions, each representing an angle in the 3D pavement, as shown in Table 3.

Table 3 Mapping from two-dimensional images to three-dimensional pavement

\begin{tabular}{|c|c|c|c|}
\hline $\begin{array}{c}\text { The angle in } \\
\text { two - dimensional }\end{array}$ & $\begin{array}{c}\text { The angle in } \\
\text { three-dimensional }\end{array}$ & $\begin{array}{c}\text { The angle in } \\
\text { two - dimensional }\end{array}$ & $\begin{array}{c}\text { The angle in } \\
\text { three-dimensional }\end{array}$ \\
\hline $0 \sim 20$ & 0 & $-20 \sim 0$ & 0 \\
\hline $20 \sim 50$ & 30 & $-50 \sim-20$ & -30 \\
\hline $50 \sim 85$ & 60 & $-85 \sim-50$ & -60 \\
\hline $85 \sim 115$ & 90 & $-115 \sim-85$ & -90 \\
\hline $115 \sim 135$ & 120 & $-135 \sim-115$ & -120 \\
\hline $135 \sim 160$ & 150 & $-160 \sim-135$ & -150 \\
\hline $160 \sim 180$ & 180 & $-180 \sim-160$ & -180 \\
\hline
\end{tabular}

Using the mapping relationship in the template library, the error can be directly eliminated by the two-dimensional image concerning that the error can meet the requirements of the crawl.

\section{Conclusion}

A localization and angle measurement system of the traffic cone is designed from the perspective of digital image processing in order to solve the problem how can the traffic cone cart identify and 
locate the traffic cones in practical work. This system can obtain images of the road surface when it is working and identify if there are traffic cones on the road. The system will locate the traffic cones after identification. We can make sure that this system can do well in traffic cone identification and localization efficiently after verification by the simulation experiments. This system also has nice scalability, it can be used in any other workings where need color recognition and distance measurement.

\section{References}

[1] ZHANG, Jinxi; KONG, Xiangjie; HAN, Dingding; WANG, Chao. Prediction Model of Asphalt Pavement PCI of Expressway Under Different Maintenance Conditions [J]. Journal of Beijing University of Technology , 2016, (1): 74-80

[2] ZHANG, Xiuwei. The Traffic Road Cones Automatic placing and retrieving System Design and Research [D]. Nanjing: Nanjing University of Science and Technology, 2013.

[3] Xinbo, Gao; Wen, Lu; Dacheng, Tao; Xuelong, Li. Image quality assessment based on multiscale geometric analysis[J]. IEEE Transactions on Image Processing , 2009, 18(7): $1409-1423$

[4] ZHAO, Shujun; DUAN, Shaoli; ZHANG, Xiaofang; LI, Lei; LIU, Xiaomin. Research on Self Calibration Method of Zoom Camera [J]. Journal of Zhengzhou University(Engineering Science), 2016, 37(5): 91-96

[5] JIN, Yuan; MENG, Jin-bao; WANG, Kun; CAI, Han-hui; XI, Ying; ZHU, Jin. The Research of Plane Array Camera Geometric Distortion Correction Method [J]. Microelectronics \& Computer, 2011, 28(10): 36-38

[6] ZHANG, Guo-quan; LI, Zhan-ming; LI, Xiang-wei; WEI, Wei-yi. Research on color image segmentation in HSV space [J]. Computer Engineering and Applications , 2010, 46(26): 179-181

[7] Habib, Ammari; Hyeonbae, Kang; Eunjoo, Kim; Mikyoung, Lim; Kaouthar, Louati. A direct algorithm for ultrasound imaging of internal [J]. SIAM Journal on Numerical Analysis , 2011, 49(3): 1177-1193

[8] Yi, Zhang; Kai, Lu; Kai, Xu; Yinghui, Gao; Richard, Wilson. Local feature point extraction for quantum images [J]. Quantum Information Processing, 2015, 14(5): 1573-1588

[9] Chia-Yen Lee; Hao-Jen Wang; Chung-Ming Chen; Ching-Cheng Chuang; Yeun-Chung Chang; Nien-Shiang Chou. A Modified Harris Corner Detection for Breast IR Image [J]. Mathematical Problems in Engineering, 2014 Vol.2014: 1024-1030

[10]Jia, Songmin; Wang, Ke; Li, Xiuzhi; Xu, Tao. Monocular Camera Three Dimensional Reconstruction Based on Variation Model [J]. Acta Optica Sinica, 2014, 34(4): 172-178 